\title{
Two ancient genome duplication events shape diversity in Hibiscus L. (Malvaceae)
}

Jonna Sofia Eriksson ( $\nabla$ jonna.eriksson@bioenv.gu.se )

Goteborgs Universitet https://orcid.org/0000-0002-1303-730X

Christine D. Bacon

Department of Biological and Environmental science

Dominic J. Bennett

Department of biologial and environmental sciences

\section{Bernard E. Pfeil}

Department of Biological and Environmental Sciences

\section{Bengt Oxelman}

Department of biological and environmental science

\section{Alexandre Antonelli}

Royal Botanic Gardens Kew

\section{Research article}

Keywords: Ancient genome duplication, gene copy, haplotype, Hibiscus, Malvaceae, paralogy, polyploidy

Posted Date: November 16th, 2020

DOl: https://doi.org/10.21203/rs.3.rs-48002/v2

License: (c) (i) This work is licensed under a Creative Commons Attribution 4.0 International License.

Read Full License 


\section{Abstract}

Background: The great diversity in plant genome size and chromosome number is partly due to polyploidization (i.e., genome doubling events). The differences in genome size and chromosome number among diploid plant species can be a window into the intriguing phenomenon of past genome doubling that may be obscured through time by the process of diploidization. The genus Hibiscus L. (Malvaceae) has a wide diversity of chromosome numbers and a complex genomic history. Hibiscus is ideal for exploring past genomic events because although two ancient genome duplication events have been identified, more are likely to be found due to its diversity of chromosome numbers. To reappraise the history of whole genome duplication events, we tested a series of scenarios describing different polyploidization events.

Results: Using target sequence capture, we generated 87 orthologous genes from four diploid species. We detected paralogues in $>54 \%$ putative single-copy genes. 34 of these genes were selected for testing three different genome duplication scenarios using gene counting. Species of Hibiscus shared one genome duplication with $H$. syriacus and one whole genome duplication occurred along the branch leading to $H$. syriacus.

Conclusions: Here, we corroborated the independent genome doubling previously found in the lineage leading to $\mathrm{H}$. syriacus and a shared genome doubling of this lineage and the remainder of Hibiscus. Additionally, we found a previously undiscovered genome duplication shared by the /Pavonia and /Malvaviscus clades (both nested within Hibiscus) with the occurrences of two copies in what were otherwise single-copy genes. Our results highlight the complexity of genomic diversity in some plant groups, which makes orthology assessment and accurate phylogenomic inference difficult.

\section{Introduction}

Whole-genome duplication (WGD), defined as the doubling of an entire genome (Jiao et al. 2012), is a well-known phenomenon in eukaryotes and is especially prevalent in plants (Stebbins 1947; Stebbins Jr 1950; Grant 1981; Otto and Whitton 2000; Soltis et al. 2009; Wendel 2015; Kim et al. 2017). Genomic studies in plants have demonstrated multiple WGD events throughout angiosperm evolution (Wendel and Cronn 2003; Cannon et al. 2006; Tuskan et al. 2006; Jaillon et al. 2007; Tang et al. 2008; Fawcett et al. 2009; Soltis et al. 2015; Landis et al. 2018) and c. $15 \%$ of all angiosperm speciation events are considered to be of polyploid origin (Wood et al. 2009). Polyploidy causes a great diversity in genome size and chromosome numbers, which can vary considerably even within families and genera (Wanscher 1934; Pellicer et al. 2018). With the increased availability of high-throughput DNA sequence data, recently formed polyploid species that arose from extant progenitor lineages have received more attention in phylogenetic studies (Buggs et al. 2012; Borrill et al. 2015). The vast amount of emerging genetic data, however, opens up potential insight into ancient polyploidization. 
The challenge of detecting ancient WGD can mainly be explained by diploidization, where polyploid genomes undergo genomic restructuring leading towards a diploid-like state (Blanc and Wolfe 2004; Soltis et al. 2015; Wendel 2015; Pellicer et al. 2018). While some loci are retained as singletons and others as duplicates, diploidization does not return the polyploid to its original diploid state (Soltis et al. 2015). Examples of mechanisms behind this are gene loss and chromosomal rearrangement (Schranz and Mitchell-Olds 2006). Moreover, mutations leading to shifts in gene expression, such as neofunctionalization and subfunctionalization, will also render the diploidized polyploid unique. Diploidization can also result from entire chromosomes being lost (aneuploidy), where synthetic polyploids have been demonstrated to suffer from an elevated chromosomal instability after genome duplication (Soltis et al. 2015). Apart from diploidization, fractionation can result in losses of entire chromosomes and copies of gene pairs duplicated through polyploidy (homoeologs). These can occur randomly with respect to either parental genome, but in some cases, losses predominantly occur in one of the parental genomes (Thomas et al. 2006; Woodhouse et al. 2010; Schnable et al. 2011; Soltis et al. 2015). In a phylogenetic context gene losses can mislead species tree inference, due to mistaken orthology. Repeated cycles of polyploid formation followed by genome rearrangement (Wendel and Cronn 2003; Soltis et al. 2015) and fractionation hinder the recognition of ancient WGD (Zhang et al. 2005).

The most common methods to identify where on a phylogeny WGD events use synteny blocks, $\mathrm{K}_{\mathrm{s}}$-rates and/or phylogenetic approaches. These approaches are powerful but are limited by: a priori information from whole-genome or transcriptome sequencing (Rabier et al. 2013; Zheng et al. 2020), saturation effects in Ks-based methods which cannot detect ancient WGD events (Vanneste et al. 2012), and phylogenetic approaches that require fully bifurcating, single-labeled trees for representing the species relationships (Rabier et al. 2013). Polyploids are best represented as a species network or a multi-labeled tree (MUL-trees) where a species can occur at multiple tips (Gregg et al. 2017), representing the homoeologues or subgenomes.

Alternative WGD detection approach are gene count methods, which require a species tree where different hypotheses can be made as to where a WGD event occurred (either along a branch or at a node), together with data on how many copies a species has in different genes (genes affected by duplication). The basic assumption is that WGD events should result in species with extra gene copies than species not affected by WGD. It should be noted that this approach does not deal with the underlying process leading to genome duplication (i.e. auto- or allopolyploidization). In addition, copies that are not linked to WGD but instead arise from single gene duplications are included in this approach, with rates of birth and loss of copies parameterized. Target sequence capture together with gene counting methods can complement $\mathrm{K}_{\mathrm{s}}$-rates, synteny and gene tree mapping-based methods that rely heavily on genome and transcriptome data.

A high diversity of recent ploidy levels and a wide range of haploid chromosome numbers in diploids suggest that several rounds of WGD have shaped the genomic history of Malvaceae s.l. subfamily Malvoideae (Menzel 1966; Bates 1969; Menzel and Wilson 1969; Bates and Blanchard Jr 1970; Fryxell 1999; Pfeil et al. 2004). For example, in cottons, Gossypium L., multiple instances of genome duplication 
have been inferred, indicating that diploid cottons are paleopolyploids (Wendel and Cronn 2003).This hypothesis was first suggested in the early 20th century through studies of chromosome pairing during meiosis (Lawrence 1931; Davie 1933) and supported by recent DNA sequencing (Wendel and Cronn 2003; Kim et al. 2017). The haploid chromosome number of $x=13$ is understood to be derived from seven homologous chromosome pairs in an ancestral cotton, which may be as old as 20-40 million years (Lawrence 1931; Davie 1933; Seelanan et al. 1997; Wendel and Cronn 2003). Regardless, the paleopolyploidization has been suggested to be even more ancient than currently understood, predating the origin of Malvaceae itself (Wendel and Cronn, 2003). Further, two ancient genome duplications were found in the genome history of cotton (Wang et al., 2012). One of the duplication events took place within the lineage Gossypium itself, while the other duplication event supports the evidence of a whole-genome multiplication (at least two WGD in short succession; Jiao et al. 2012) shared by all eudicots (Wang et al. 2012).

Hibiscus $\mathrm{L}$. is a widely cultivated genus of Malvaceae, characterized by its numerous rounds of polyploidy (Wilson 1994; Pfeil et al. 2004; Kim et al. 2017). The taxonomic delimitation of Hibiscus has been unstable (Pfeil et al. 2005 and references therein) with nuclear and chloroplast genes suggesting the traditional circumscription is a paraphyletic group. Phylogenetic work showed that traditionally-defined Hibiscus includes representatives of other genera that had been classified in Hibisceae, Malvavisceae (including e.g., Pavonia) and Decaschistieae tribes (Pfeil et al. 2002). Pfeil and Crisp (2005) proposed to treat the three tribes under Hibiscus s.l., which we apply here. Within this classification, unranked clade names preceeded by a forward slash (/) are used to indicate clades nested within Hibiscus sensu Pfeil and Crisp (2005). Note that not all combinations at the species level have been made in that classification, so we use existing binomials in other genera as necessary.

The diversity of haploid chromosome numbers in Hibiscus may reflect ancient genome doubling events followed by diploidization. A group of species within Hibiscus, clade /Furcaria, is a well-studied group of polyploids (Wilson 1994, 2006). Menzel (1966) proposed that the diploid Hibiscus cannabinus L. in /Furcaria, with a haploid chromosome number of $x=18$, may have been derived through ancient WGD events with a base chromosome number of either six or nine. Hibiscus section /Euhibiscus has a base chromosome number of $x=20-22$ (e.g. H. rosa-sinensis and H. syriacus; Skovsted, 1941). In addition, the mostly Neotropical clade /Pavonia, is hypothesized to originate from $x=7$ or 14 based on the multiples of chromosome counts inferred by several species (Fryxell, 1999, treated under Pavonia). Only 29 Pavonia species of the c. 220 have been counted (Fryxell, 1999 and references therein; Davie 1933; Fernández et al. 2003). Of these, two are $2 n=28,23$ are $2 n=56$, and two are $2 n=112$, indicating that many of the species are likely to be higher polyploids.

Two ancient genome doubling events followed by diploidization were identified in the $H$. syriacus $L$. lineage (clade /Euhibiscus; Kim et al. 2017). The two WGD events are considered to be independent and took place after the divergence from the H. syriacus-G. raimondii common ancestor (Kim et al. 2017). The varying haploid chromosome numbers within Hibiscus and between the sister genus Gossypium, may reflect various degrees of diploidization, with chromosome fusion/fission in different lineages after 
speciation. Whether diploidization is the underlying cause for the diverse base chromosome number found in species of Hibiscus is yet to be understood.

In this study we determine if diploid and polyploid species of Hibiscus has signatures of ancient genome duplications, and if these are shared with the WGDs found in $H$. syriacus. Based on previous phylogenetic hypotheses (Pfeil et al. 2002; Baum et al. 2004; Pfeil et al. 2004), and base chromosome number variation between clades in Hibiscus, we present three hypothetical scenarios (Figure1; scenario S1-S3) that illustrate the likely genomic origins of Hibiscus before diploidization using the two WGD events detected previously in $\mathrm{H}$. syriacus (Figure 1). To test amongst these hypotheses we use diploid members of Hibiscus clade /Furcaria that are assumed to be derived from an ancient genome duplication (Menzel 1966). We furthermore select a species from /Pavonia, given the lack of diploids in this group, their relatively high chromosome numbers $(2 n=56-112$; Fryxell, 1999 and references therein) and the unknown base chromosome number. In the first scenario, only $H$. syriacus show evidence of two whole-genome duplications (S1; figure 1a). However, considering that the base chromosome numbers varies greatly within Hibiscus (e.g. Hibiscus section Trionum $x=7$ or 14 (Fryxell, 1999), section Furcaria $x=18$ (Skovsted 1941) and /Euhibiscus $x=20-22$ (e.g. H. rosa-sinensis and H. syriacus; Skovsted, 1941) - the WGD events leading to $H$. syriacus (S1) may include other species of Hibiscus. In the second scenario, we explore if one of the WGD events in H. syriacus is shared by all species of Hibiscus, and if the second duplication is restricted to $H$. syriacus (S2; Figure $1 b$ ). In the third scenario, we test whether both WGD events in H. syriacus are shared by all species of Hibiscus (S3; Figure 1c).

Here, we develop a new analytical framework to identify multiple haplotypes and assemble them into full sequences. Current methods use different approaches to overcome the challenge of connecting alleles/homoeologues/haplotypes by using ambiguity codes where the read depth is too shallow to connect two variants (Kates et al. 2018) or by using a known pedigree (Browning and Browning, 2011; Martin et al. 2016). Alternative approaches use a reference genome or construct a de novo reference from read data. However, these algorithms are built on the assumptions that all organisms are diploids and that only two haplotypes exist at a locus. In the presence of more than two haplotypes, such as in polyploid plants, either chimeric haplotypes are produced or the number of haplotypes is underestimated. Our approach makes no assumption regarding the number of sequence copies or ploidy level and does not construct chimeric sequences as a result of more than two copies found in polyploid species.

\section{Results}

Target capture, mapping and paralogue assembly - Data from six individuals were successfully sequenced with a mean number of 1,261,242 reads per individual after trimming [Additional file 1]. Out of 87 genes targeted, 14 genes had one sequence copy per species (referred here as SCG) and 20 genes showed more than one sequence copy per species (termed here as MCG). All genes had contigs that were overlapping for the same region for all species. The mean read depth (coverage) of each assembly ranged between 81-413 (Table 1). The final alignments had a mean length of 1,972 bp (ranging between 934-3151 bp). 
Table 1. Mean read depth across all base pairs per species per gene. The average read coverage is calculated across all contigs and per exons for each gene. Asterisk $\left(^{*}\right)$ refer to genes that have only one sequence copy per species (e.g. single copy genes).

\begin{tabular}{|c|c|c|c|c|c|c|}
\hline \multirow[b]{2}{*}{ Gene } & \multicolumn{5}{|c|}{ Mean read depth (coverage across all assemblies) per locus } & \multirow[b]{2}{*}{ P. triloba } \\
\hline & H. cannabinus 1 & H. cannabinus 2 & H. cannabinus 3 & H. mechowii & H. trionum & \\
\hline $\mathrm{ABC}-\mathrm{C} 2$ & 206 & 120 & 180 & 324 & 66 & 53 \\
\hline ACCS & 231 & 179 & 172 & 284 & 42 & 38 \\
\hline Acylamino & 480 & 300 & 330 & 823 & 122 & 76 \\
\hline AglucanP & 411 & 638 & 597 & 399 & 171 & 112 \\
\hline Ankyrin & 239 & 151 & 145 & 315 & 91 & 77 \\
\hline Bgalactosidase8 & 256 & 193 & 178 & 322 & 57 & 70 \\
\hline CAD & 406 & 295 & 270 & 381 & 60 & 57 \\
\hline Calcium11 & 494 & 293 & 315 & 600 & 75 & 158 \\
\hline Calcium-ATPase1 & 215 & 195 & 196 & 287 & 73 & 72 \\
\hline Calcium-ATPase3* & 338 & 217 & 243 & 460 & 87 & 76 \\
\hline Callose* & 93 & 84 & 222 & 91 & 42 & 45 \\
\hline CesA1 & 200 & 209 & 147 & 255 & 79 & 116 \\
\hline DEAD-ATP* & 324 & 308 & 284 & 150 & 87 & 90 \\
\hline EIF-2B & 133 & 96 & 98 & 160 & 23 & 35 \\
\hline $\mathrm{F} 5 \mathrm{H}$ & 232 & 200 & 172 & 268 & 66 & 52 \\
\hline Formin2 & 141 & 117 & 109 & 151 & 31 & 27 \\
\hline Glutamine & 425 & 302 & 349 & 744 & 158 & 85 \\
\hline GPDH & 297 & 264 & 258 & 459 & 136 & 100 \\
\hline Importin4* & 413 & 271 & 328 & 538 & 55 & 109 \\
\hline Kinesin-KCA2* & 268 & 233 & 276 & 291 & 76 & 87 \\
\hline Kinesin-Kp1 & 333 & 410 & 398 & 473 & 106 & 44 \\
\hline LOC105792102 & 187 & 153 & 137 & 221 & 58 & 58 \\
\hline MAP3K & 385 & 280 & 274 & 441 & 102 & 78 \\
\hline Mechanosensitive* & 481 & 467 & 481 & 622 & 195 & 143 \\
\hline MNS4* & 299 & 276 & 220 & 353 & 91 & 83 \\
\hline NF-X1-zinc* & 274 & 282 & 271 & 429 & 100 & 85 \\
\hline Oxysterol-1D* & 1103 & 1224 & 1095 & 2002 & 323 & 316 \\
\hline Phospholipase & 212 & 178 & 178 & 299 & 62 & 65 \\
\hline Plasma-ATPase & 216 & 189 & 174 & 238 & 73 & 56 \\
\hline Polysub2* & 428 & 562 & 220 & 798 & 170 & 161 \\
\hline RRP5* & 404 & 307 & 311 & 410 & 103 & 114 \\
\hline SBT3-5* & 591 & 467 & 451 & - & 136 & 102 \\
\hline SD1-1* & 628 & 495 & 524 & 685 & 127 & 116 \\
\hline TGH* & 743 & 560 & 570 & 405 & 172 & 162 \\
\hline Mean coverage across all loci & 312 & 261 & 259 & 371 & 87 & 80 \\
\hline
\end{tabular}

Occurrence of paralogous genes - Despite targeting low-copy nuclear genes (from transcriptome at hand; Hibiscus cannabinus, $1 \mathrm{KP}$ Code OLXF) we found that $54 \%$ of the genes contained more than the two 
variants (i.e. haplotypes) found in one of the diploid H. cannabinus accessions (i.e. H. cannabinus 1 ). The GPDH gene had ten different DNA sequence variants in $H$. cannabinus 1 (our sequenced individual), but only a single variant was found in the $H$. cannabinus transcriptome. However, this gene appeared at eight locations in the G. raimondii genome. The Glutamine gene (LOC 105766 149) with three H. cannabinus1 variants was only found at one location in the transcriptome and also appeared as a single copy in the $G$. raimondii genome. We consistently observed subtrees that had either one or two or more sequence copies from $H$. cannabinus (Table 2). Hibiscus syriacus was often seen to have more than three copies in each subtree, whereas the /Pavonia clade species nearly always had twice as many copies as seen in $H$. cannabinus.

Table 2. Gene count data used for likelihood scenario testing. Duplicated gene name represents separate paralogous clades. The number of copies were counted for each gene as the number of sequences from one individual in a clade that had Gossypium (G) as an outgroup. The abbreviations are short for Hibiscus syriacus (S), H. cannabinus (C), H. mechowii (W), H. trionum (T) and Pavonia triloba $(\mathrm{P})$. 


\begin{tabular}{|c|c|c|c|c|c|c|}
\hline \multirow[b]{2}{*}{ Gene name } & \multicolumn{6}{|c|}{ Number of copies } \\
\hline & G & $\mathrm{S}$ & $\mathrm{C}$ & M & $\mathrm{T}$ & $\mathrm{P}$ \\
\hline $\mathrm{ABC}-\mathrm{c} 2$ & 1 & 2 & 1 & 1 & 0 & 0 \\
\hline ABC-c2 & 1 & 1 & 1 & 0 & 1 & 3 \\
\hline ACCS & 1 & 0 & 2 & 1 & 0 & 2 \\
\hline ACCS & 1 & 3 & 2 & 2 & 2 & 1 \\
\hline Acylamino & 1 & 3 & 3 & 2 & 2 & 5 \\
\hline AglucanP & 1 & 9 & 2 & 2 & 2 & 12 \\
\hline Ankyrin & 3 & 6 & 2 & 2 & 2 & 4 \\
\hline Ankyrin & 1 & 1 & 2 & 0 & 0 & 0 \\
\hline Bgalactosidase 8 & 1 & 3 & 2 & 2 & 6 & 4 \\
\hline CAD & 4 & 2 & 3 & 2 & 2 & 3 \\
\hline CAD & 1 & 1 & 1 & 1 & 1 & 1 \\
\hline Calcium11 & 1 & 4 & 2 & 2 & 0 & 3 \\
\hline Calcium11 & 2 & 5 & 2 & 3 & 6 & 2 \\
\hline Calcium-atpase 1 & 1 & 3 & 1 & 1 & 1 & 2 \\
\hline Calcium-atpase1 & 1 & 0 & 1 & 1 & 1 & 2 \\
\hline CesA1 & 3 & 4 & 2 & 2 & 2 & 4 \\
\hline EIF-2B & 2 & 2 & 1 & 1 & 1 & 2 \\
\hline EIF-2B & 1 & 2 & 1 & 1 & 1 & 2 \\
\hline $\mathrm{F} 5 \mathrm{H}$ & 1 & 1 & 1 & 1 & 1 & 2 \\
\hline $\mathrm{F} 5 \mathrm{H}$ & 1 & 4 & 2 & 2 & 2 & 4 \\
\hline $\mathrm{F} 5 \mathrm{H}$ & 1 & 0 & 2 & 2 & 1 & 2 \\
\hline Formin2 & 1 & 5 & 1 & 1 & 0 & 0 \\
\hline Formin2 & 1 & 3 & 2 & 2 & 2 & 2 \\
\hline Formin2 & 4 & 7 & 2 & 3 & 2 & 2 \\
\hline Glutamine & 1 & 5 & 3 & 3 & 2 & 1 \\
\hline GPDH & 1 & 2 & 1 & 0 & 0 & 2 \\
\hline GPDH & 1 & 1 & 2 & 1 & 1 & 1 \\
\hline
\end{tabular}




\begin{tabular}{|l|l|l|l|l|l|l|} 
GPDH & 1 & 2 & 2 & 2 & 2 & 3 \\
\hline GPDH & 1 & 6 & 2 & 2 & 2 & 2 \\
\hline GPDH & 2 & 10 & 1 & 2 & 2 & 4 \\
\hline Kinesin-KP1 & 1 & 2 & 1 & 1 & 1 & 2 \\
\hline Kinesin-KP1 & 3 & 2 & 1 & 1 & 1 & 2 \\
\hline LOC105792102 & 2 & 2 & 1 & 1 & 1 & 2 \\
\hline LOC105792102 & 1 & 3 & 2 & 2 & 2 & 2 \\
\hline LOC105792102 & 1 & 2 & 2 & 2 & 2 & 4 \\
\hline LOC105792102 & 1 & 7 & 2 & 2 & 1 & 0 \\
\hline LOC105792102 & 1 & 1 & 1 & 1 & 1 & 3 \\
\hline MAP3K & 1 & 4 & 1 & 1 & 1 & 2 \\
\hline MAP3K & 1 & 3 & 2 & 2 & 2 & 4 \\
\hline Phospolipase & 1 & 1 & 1 & 1 & 0 & 0 \\
\hline Phospolipase & 1 & 4 & 2 & 2 & 0 & 0 \\
\hline Phospolipase & 1 & 2 & 2 & 0 & 0 & 0 \\
\hline Plasma-ATPase & 1 & 2 & 1 & 1 & 0 & 0 \\
\hline Plasma-ATPase & 1 & 7 & 2 & 3 & 2 & 0 \\
\hline
\end{tabular}

Phylogenetic inference - For the single-copy gene trees (SCG), 10 out of 14 genes showed the same topological relationships with the /Furcaria clade species forming a clade sister to $H$. trionum $+P$. triloba, and this larger clade in turn sister to H. syriacus (Figure 2), consistent with Pfeil and Crisp (2005). The other four genes often had an extra gene copy from one taxon appearing in a different relationship indicating either a deep coalescence event or another paralogous copy (e.g. gene Oxysterol-D1; Suppl. Figure 1). The phylogenetic trees and subtrees (paralogous clades within one gene tree) strongly support a relationship previously reported (Pfeil et al. 2002), with $H$. syriacus sister to /Furcaria $+(H$. trionum $+P$. triloba). In most gene trees, multi-copy genes (MCGs) and SCGs likewise, species of Pavonia possessed at least two copies that form a clade (Suppl. Figure 2-21).

Scenario testing - A species tree was generated to test amongst three genome evolution hypotheses using the WGDgc R package (Rabier et al. 2013). All parameters had an ESS value $>200$ indicating that the priors had all converged and a maximum clade credibility tree was created summarizing the clade posterior probabilities on a single tree. The gene count data consisted of 44 data points (subtrees) over 20 MCG (Table 2). The rates of duplication and loss were estimated to be 0.03 and 0.003 , respectively. 
The scenario testing using gene count data showed that S2 (one shared genome duplication with $H$. syriacus and one WGD contained within $H$. syriacus) was the preferred model given the observed distribution of paralogous gene copies in Hibiscus (Figure 2). We found the null-scenario (no WGD events) to be the least likely model to explain the data among the models we evaluated. Through the process of identifying paralogous copies and constructing the gene count data, we found that: (1) /Pavonia species had twice as many copies as /Furcaria species and (2) the presence of MCG and SCG were congruent with the occurrence of two gene copies within /Pavonia species. Our results thus indicate a third WGD event. All the scenarios had a lower likelihood score with the inclusion of a third WGD event and the preferred scenario $(S 2 ; \omega A I C>0.95)$ did not change with the inclusion of a third WGD in the clade /Pavonia (Table 3).

Table 3. Log-likelihood scores, AIC and weighted AIC from gene count data on each scenario. Two setups were tested for the three hypothetical WGD scenarios. The first setup tested two WGD events on the three scenarios; S1 where two WGDs are found in $H$. syriacus; S2 where one WGD is shared by all species of Hibiscus; and S3 were both WGD are shared by all species of Hibiscus. The null hypothesis tests whether no WGD has occurred in Hibiscus. The last setup tested an additional WGD within /Pavonia, following the same S1, S2, and S3 scenarios.

\begin{tabular}{|c|r|l|r|r|r|}
\hline 2 WGD & loglikelihood & AIC & deltaAIC & rel.LL & AIC weights \\
\hline S1 & -294.4646 & 596.9292 & 8.2118 & 0.01647 & 0.0162 \\
\hline S2 & -290.3586 & 588.7173 & 0 & 1 & 0.9833 \\
\hline S3 & -297.9675 & 603.9350 & 15.2176 & 0.0005 & 0.0005 \\
\hline null & -303.7357 & 611.4714 & 22.7540 & $1.1455 \mathrm{e}-05$ & $1.1264 \mathrm{e}-05$ \\
\hline 3 WGD & & & & & \\
\hline S1 & -276.8404 & 563.6808 & 13.5126 & 0.0012 & 0.0012 \\
\hline S2 & -270.0841 & 550.1682 & 0 & 1 & 0.9988 \\
\hline S3 & -285.7683 & 581.5366 & 31.3684 & $1.5432 \mathrm{e}-07$ & $1.5414 \mathrm{e}-07$ \\
\hline null & -303.7357 & 611.4714 & 61.3032 & $4.8771 \mathrm{e}-14$ & $4.8714 \mathrm{e}-14$ \\
\hline
\end{tabular}

\section{Discussion}

While it is widely accepted that recent polyploids originate through complex evolutionary histories, diploid species also often have complicated genomes, preventing an accurate phylogenetic inference. In this study we present evidence that the evolution of Hibiscus includes several whole genome duplication (WGD) events. Even diploid species (i.e. not subject to recent polyploidy) - H. cannabinus and $H$. mechowii - contained additional copies of genes that were expected to be single copy. Taken together, evidence is consistent with ancient duplications (that duplicate many genes) and the retention of many of these gene lineages, despite a return to diploid genetic state.

We found that whole-genome duplication events best explain the observed number of sequences in Hibiscus. The null-hypothesis - where it is assumed that no WGD events took place in Hibiscus - had the lowest likelihood compared to the alternative scenarios. Consequently, single gene duplications are a less 
likely explanation than WGD for the occurrence of multiple gene copies found within subtrees. Instead, we found that S2 (one genome duplication shared by all Hibiscus species and one genome duplication leading to $H$. syriacus), best explained the pattern observed in the trees/gene count data. Within each gene subtree (defined by one Gossypium copy as outgroup) H. syriacus possess on average twice as many copies as /Furcaria species, indicating an independent genome duplication leading to $\mathrm{H}$. syriacus consistent with the chromosome number $(2 n=40$ (Skovsted 1941). We corroborate the previous findings of two WGDs in H. syriacus (Kim et al. 2017), but with one modification; one of the duplication events is older than previously presumed (by Kim et al. 2017) and had already occurred somewhere along the branch leading to Hibiscus.

\section{An Additional Recent Polyploid Event in Pavonia's Past}

During the process of identifying sequence copies (alleles and paralogues) we found that /Pavonia, within clade /Trionum, always possessed twice as many copies relative to the other species in the clade (i.e. H. trionum in our sample). Furthermore, /Pavonia also possessed twice as many copies as /Furcaria, the sister clade to /Trionum, suggesting that a recent genome duplication occurred in /Pavonia. By including a third genome duplication in our scenario testing, we clearly show that part of the data can be explained by an independent genome duplication in /Pavonia. All three scenarios resulted in lower loglikelihood scores when three WGD events were included.

The inferred base chromosome number in /Pavonia - either $n=7$ or 14 - reflects the uncertainty of the genomic history (Fryxell, 1999). Here, we found that $P$. triloba underwent a separate genome duplication in addition to the shared one with all species included in Hibiscus. However, whether it is a recent duplication within P. triloba or a duplication shared with other related species (/Pavonia and /Trionum) cannot be determined here. We infer from our results that the base chromosome number in /Pavonia and /Trionum is likely to be $n=14$ and not $n=7$ (Fryxell, 1999; Les 2017), due to the shared genome duplication with all species in Hibiscus. This hypothesis is also supported by the lack of "diploid" species in Pavonia with $2 n=14$ (Fryxell, 1999), if seven is the true haploid chromosome number. No other species in /Trionum have been reported to have $2 n=14$ chromosomes. On the other hand, $2 n=28$ counts and above have been found $H$. trionum (Murray et al. 2008), Malvaviscus arboreus (incl. in /Pavonia; Turner and Mendenhall 1993) and in Pavonia species.

Additional copies were found within some of the paralogous genes (Suppl. Figs. 3 - 4, 10, 13) that may either be relicts of older genome duplication events or the consequence of gains of extra copies through independent gene duplication. For example, the Acylamino gene (Suppl. Figure 5) has a third clade consisting of species from /Furcaria and /Pavonia but lack copies from H. syriacus and Gossypium. These additional copies suggest an independent gene duplication, or losses of copies in Gossypium and $H$. syriacus. Furthermore, in the same gene we find two clades containing $H$. cannabinus gene copies sister to its close relative $H$. mechowii consistent with gene duplication restricted to $H$. cannabinus. These gains and losses of copies are common throughout all the genes and may reflect processes such as 
independent gene duplications and losses of copies through fractionation or diploidization complicating an already complex history.

\section{Data quality}

The challenge of separating alleles and copies during sequence read assembly is a crucial one for the success of this study. Current methods typically assume that organisms are diploids and thus can only have two haplotypes at a locus (Browning and Browning 2011). These assumptions are violated in the presence of more than two haplotypes, such as in polyploid and paleopolyploid plants, where current methods may produce either chimeric haplotypes or an underestimate of the number of haplotypes. Chimeric sequences can also arise through tandem duplications. Cluster analysis is a methodological advance because it identifies the possible number of copies that had been sequenced in the sample, if the sequence copies are distinct from each other in the exon regions (or any used reference region). In contrast, using tools that produce maximum two haplotypes/alleles (e.g. Eriksson et al. 2017), we found that most of the copies were not identified and information was lost. One caveat with this approach in this study, however, is the possibility of underestimating the number of copies - sequence copies that we miss due to conserved exon regions, but may have nucleotide differences in the intron regions. While this approach can tease apart distinct haplotypes, it does not separate allelic variants when the polymorphic sites connecting two alleles are too far apart (further away than two paired-end reads can overlap). Thus, possible allelic variants likely have been overlooked in this study as it continues to be impossible to separate variants with current methods.

\section{Conclusions}

Problems with identifying paralogues, homoeologues and allelic variants has negative implications on understanding polyploidy and the processes of diploidization, a common feature found in plants. Previous studies rely on whole genome data to discover ancient genome duplications. We demonstrate here that target sequence capture of a relatively small number of loci can complement existing methods for resolving WGD events. With the information from gene trees and gene count data, new insights into genome duplication were found in diploid and polyploid species of Hibiscus. Furthermore, by considering the variation of base chromosome number seen between clades in Hibiscus there are potentially other genome duplications that we have not corroborated in this study. Our results also highlight that even diploid species have complex genomes and that there may be a vast number of diploid species that contain traces of ancient WGDs in other plant groups. Considering the diverse chromosome numbers in plants, more evidence of ancient genome duplications and processes of diploidizations are yet to be uncovered.

\section{Materials And Methods}

Sampling and DNA extraction - Species with known ploidy were selected to reappraise possible genome duplications in Hibiscus (Additional file 1). Two diploid species, with three specimens of H. cannabinus L. 
and one $H$. mechowii Garcke (both $2 n=32$ ) were selected from clade /Furcaria (C and M in Figure 1): Pavonia triloba Hochst. ex. Webb (clade /Pavonia within Hibiscus) with unknown chromosome number (P in Figure 1: H. trionum L. from clade /Trionum, a diploid/tetraploid species $(2 n=28,56$; Dasgupta 1981 ; $T$ in Figure 1); and two species from previous whole genome sequencing studies, $H$. syriacus from clade /Euhibiscus (GenBank assembly accession GCA_001696755.1; Kim et al. 2017) and Gossypium raimondii, the latter not being part of Hibiscus (GenBank assembly accession GCF_000327365.1, Paterson et al. 2012; Xu et al. 2013). Silica dried leaves were collected and DNA was extracted from 25-30 $\mathrm{mg}$ of plant material using DNeasy Plant mini Kit (Qiagen, Valencia, CA, USA) with two deviations from the manufacturer's protocol: supernatant with AP1 buffer was incubated at $42^{\circ} \mathrm{C}$ for $24 \mathrm{~h}$, and a $30 \mathrm{~min}$ incubation with AW1 buffer. Samples with excess secondary compounds (polysaccharides) had an additional volume of AP1 buffer added to reduce the viscosity. Samples that discolored the column membrane (e.g. phenol contaminants) incurred an additional step of cleaning with $95 \%$ ethanol. Only samples with high quality DNA with an absorbance ratio falling within 1.8-2.0 (260/280 nm and 230/260 $\mathrm{nm}$ ) were used for the downstream workflow.

Library preparation - Genomic DNA was sheared using Covaris S220 instrument (Covaris, Woburn, Massachusetts, USA) to a fragment size between 600-800 bp and end-repaired with library NEXTflex rapid DNA-Seq kit (BIOO Scientific, Austin, Texas, USA). End-repaired fragments were barcoded using NEXTflex DNA Barcodes and size selected to optimize recovery of fragments between 600-800 bp using Ampure XP beads according to the manufacturer's protocol. A polymerase chain reaction (PCR) was performed using master mix and primer mix provided in the library kit, with the cycling programme: $98^{\circ} \mathrm{C}$, $2^{\prime} ; 14 x\left(98^{\circ} \mathrm{C}, 30^{\prime \prime} ; 65^{\circ} \mathrm{C}, 30^{\prime \prime} ; 72^{\circ} \mathrm{C}, 60^{\prime \prime}\right) ; 72^{\circ} \mathrm{C}, 4^{\prime}$. PCR products were purified with $0.4 x$ Agencourt AMPure $\mathrm{XP}$ beads (Beckman Coulter) and eluted in $20 \mu \mathrm{l}$ resuspension buffer.

Target capture and sequencing - Target gene capture was performed using custom made MYbaits (MYcroarray, Ann Arbor, Michigan), targeting 87 low-copy nuclear genes, designed using the Hibiscus cannabinus transcriptome (Johnson et al. 2012; Matasci et al. 2014; Wickett et al. 2014; Xie et al. 2014) annotated using the Gossypium raimondii genome (Paterson et al. 2012; Xu et al. 2013). Probes were selected from regions with exon lengths $>90 \mathrm{bp}$ and intron lengths $<1,000 \mathrm{bp}$. Selected exons were blasted against the $G$. raimondii genome using NCBI megablast with an e-value of 10 (a high e-value was chosen to look for distant homologues between $\mathrm{H}$. cannabinus and $\mathrm{G}$. raimondii). Only regions with a single copy in the transcriptome and a nucleotide similarity of above $86 \%$ to the Gossypium genome were accepted.

Six NEXTFlex barcoded libraries were pooled per capture reaction following the protocol from the manufacturer. Each pooled reaction was incubated at $65^{\circ} \mathrm{C}$ and incubated for $24 \mathrm{~h}$. For libraries prepared from silica dried material incubation was performed for $16 \mathrm{~h}$. Targeted DNA was captured and purified using Dynabeads MyOne Streptavidin C1 beads (Invitrogen Dynal AS, Oslo, Norway), before PCR amplification with the following programme: $98^{\circ} \mathrm{C}, 2^{\prime} ; 14 x\left(98^{\circ} \mathrm{C}, 20^{\prime \prime} ; 65^{\circ} \mathrm{C}, 30^{\prime \prime} ; 72^{\circ} \mathrm{C}, 60^{\prime \prime}\right) ; 72^{\circ} \mathrm{C}, 5^{\prime}$. PCR products were purified using $0.4 \times$ AMPure XP beads. To remove any residue of alcohol the tubes were air dried until the beads were visibly dry (over-drying beads results in lower yield of captured PCR products) 
and eluted in $20 \mu \mathrm{l}$ resuspension buffer. Fragment size length was checked on a Tapestation 2200 (Agilent Technologies) with D1000 tapes and DNA quantity was checked on an Invitrogen ${ }^{\mathrm{TM}}$ Qubit $^{\mathrm{TM}} 3.0$ Fluorometer with HS buffer. The sequencing was performed by the SciLifeLab facility in Stockholm, Sweden, on an Illumina MiSeq (San Diego, California, USA) instrument with 300 bp paired-end reads.

Quality trimming and mapping - The reads were processed with CLC Genomic Workbench (CLC Bio, Aarhus, Denmark) to trim the barcodes and Illumina adaptors from the reads. Low quality reads were removed with a phred-score quality threshold of 20 and duplicate reads. Each sample was individually mapped to the targeted probe sequences with a similarity score of 0.7. Mapped probes were sorted using Samtools v.1.3.14 (Li et al. 2009) retaining the information of the read names and their position with respect to the probes.

We constructed a pipeline that assembles sequence copies that may be haplotypes/homoeologues, hereafter multiple variants, by mapping to the references in two steps: the first step (URL:

https://github.com/DomBennett/Project-cluster) assembles clusters of identical reads corresponding to all the captured target regions. The second step iteratively adds flanking regions where reads support, to build the original genomic sequences without joining parts of sequences together that come from different copies. The first step in the pipeline uses the SAM files and the tool CD-HIT (Li and Godzik 2006; Fu et al. 2012) to identify multiple variants by clustering similar reads. In brief, reads mapped to one of the exons are removed when found outside the exon boundaries. CD-HIT then identifies reads that are similar above a certain threshold. We used a 1.0 similarity score and a minimum length of $60 \mathrm{bp}$. If CDHIT finds a read that does not have sufficient similarity with a cluster, that read forms a new cluster. Clusters that were represented by only 10 reads or less were deleted.

The second part of the pipeline used the mapping tool in Geneious v11.1.3 (https://www.geneious.com, (Kearse et al. 2012) to reconstruct full sequences (i.e., containing both exons and introns) from the identified clusters. The exon that had the highest number of clusters was used for constructing full sequences. A consensus was made for each cluster and used as a reference sequence. We used custom settings where full reads (reads that contain both exon and intron sequence data) mapped to the reference had to be without mismatches or gaps, and a word length of 99 characters. Each assembly was iterated five times, where the consensus sequence made from each assembly served as a new reference for the next iteration. We removed copy assemblies that contained positions with polymorphic sites. This assembly step generates sequences in the form 'exon-intron-exon' connecting individual exons by adding intervening introns, unless the introns are so long that the iterations do not produce overlapping contigs. Thus, the exons that had fewer clusters were indirectly included by the 'exon-intron-exon' assembly step.

The resulting sequences were aligned using MAFFT v7.388 (Katoh et al. 2002; Katoh and Standley 2013) with the auto algorithm (selecting the appropriate method according to the size of data) and default gap penalties. For the gene alignments where sequences did not overlap - due to exons position being too far away and the selection of highest number of clusters may differ between samples -- a higher number of iterations (up to 25 times) in the assembly step could in some cases lead to the sequences spanning the 
entire gene length (all exons). Genomic data from $\mathrm{H}$. syriacus and G. raimondii were downloaded from NCBI (accession numbers GCA_001696755.1 and GCF_000327365.1, respectively). The probes from each gene were mapped to both genomes to find the location of the singletons or duplicated copies using medium sensitivity/fast settings in Geneious. Sequences from both genomes were added to the alignment using the -add option in MAFFT. Only gene alignments where the sequences overlapped the same exon or the neighboring exons were used for phylogenetic analyses, that resulted in 20 multi-copy genes (MCG, where diploid species have more than one haplotype) and nine single-copy genes (SCG, where diploids only have one haplotype). The rest of the genes were either incomplete due to missing taxa or because of non-overlapping sequences in the alignments.

In one of the gene alignments (Phospholipase), one Pavonia copy had the $5^{\prime}$ and the $3^{\prime}$ end of two sequences apparently swapped, likely due to a recombination event between two copies. We inspected the assembly in order to find any indication of chimeric mapping that could be the result of conserved regions - in which reads accidentally map to multiple copies - however no such indication could be found. In such cases we created two sequences by separating the front and back half of the recombined sequence.

Phylogenetic analysis - Bayesian inference was performed using MrBayes v3.2.6 (Ronquist and Huelsenbeck 2003) for 20 MCG and 14 SCG using a reverse model jumping Markov Chain Monte Carlo method (rjMCMC) to average over all 203 possible combinations of substitution models (Ronquist and Huelsenbeck, 2003). We allowed among site rate heterogeneity (using a gamma distribution with shape parameter alpha) for all models and genes, as we expected difference in rates between exons and introns as well as codon positions. The branch length prior (brlenpr) was set to unconstrained exponential molecular clock set to 100 , to allow for smaller branch length prior means (Marshall et al. 2006). All other options were set to program defaults. We ran each analysis on two parallel chains for two independent runs of 10 million generations, sampling every 2,000 generations. We applied a burn-in of $10 \%$ after checking convergence such that all parameters had an effective sample size (ESS) >200 with Tracer v1.6 (Drummond et al. 2012). Trees were annotated using TreeAnnotator v1.8.1 (part of the BEAST package) before being visualized in Figtree v1.4.2 (tree.bio.ed.ac.uk/software/figtree/).

A species tree was constructed for WGD scenario testing using ten SCG that contained one copy per specimen. The analysis was run under the SpeciesTreeUCLN template in BEAST2 (Bouckaert et al. 2014) with a three-rate substitution model (TR93; Tamura and Nei 1993) chosen by comparing all tracer files from all genes using a MrBayes v3.2.6 mixed model selection with a mean k-revmat of 3.12 (Huelsenbeck et al. 2004). We employed a birth-death process for the tree prior and an uncorrelated lognormal relaxed molecular clock model (Drummond et al. 2006) set to 0.0055 subs/site/Ma based on a priori information for the family (Wendel et al. 1995; Kay et al. 2006; Koopman and Baum 2008) was used. The analysis was run for 40 million generations sampling every 5000 generations. The parameters were checked for convergence in Tracer v1.6 and a burn-in of $10 \%$ of the trees was removed using TreeAnnotator in BEAST2 package. 
Species tree and scenario testingusing likelihood scores - We compared the log-likelihood scores for the observed gene copy numbers in each taxon from the 20 MCG on three WGD scenarios (Figure 1) using the WGDgc v1.2 R package (Rabier et al. 2013). WGDgc uses the number of copies across gene families (defined as a gene that contains more than two gene copies of a given taxa) inferred on a species tree. We counted the number of copies in every gene for each species that formed a clade that had at least one Gossypium copy as sister to Hibisceae (i.e., duplications that lead to or are within the Hibisceae lineage, and thus may be linked to gene duplication events). Furthermore, each gene may contain several sequence copies that form subtrees (several clades with Gossypium copies sister to Hibisceae copies) that would be each be counted as one data point in the gene count data. Extra clades that were missing a Gossypium copy were not used. The number of copies were converted into gene count data manually. We used a Dirac delta prior set to 1 for the number of copies at the root, assuming there is always a single copy present at the root. The starting values of the duplication (birth) and loss (death) rates were set to the default values according to the manual and were estimated using maximum likelihood. The type of conditioning for the likelihood calculation was set to "twoOrMore", allowing for gene families to have two or more genes. On the species tree, we fixed WGD events to the mid-point of the species tree branches according to our three scenarios (e.g. for S1 the tree will have two independent WGD events on the branch leading to $S$, and so on, as per Figure 1). Akaike weights (WAIC) are calculated by estimating the relative model likelihoods by normalizing with the sum of the likelihoods of all models (Wagenmakers and Farrell 2004) and can be interpreted as the probability that the model is the most likely given the data (gene count) and candidate models (scenarios) (Wagenmakers and Farrell 2004). R code for reproducing our analysis can be found on GitHub (https://github.com/AntonelliLab/WGD-scenario-testing-in-hibiscus).

\section{Declarations}

\section{Ethics approval and consent to participate}

Not applicable.

\section{Consent for publication}

Not applicable.

\section{Availability of supporting data}

The data set(s) supporting the results of this article is (are) available in the [repository name] repository, [unique persistent identifier and hyperlink to dataset(s) in http:// format]. (Data will be deposited before publication.)

\section{Competing interests}

The authors declare that they have no competing interests.

\section{Funding}


This work was supported by grants from the Swedish Research Council, the Royal Swedish Academy of Sciences [grant number 2009-5206], Lars Hiertas Minne fund, The Royal Physiographic Society in Lund, Helge Ax:son Johnsons fund and the Lundgrenska fund to BEP; the Swedish Research Council [grant number B0569601], the European Research Council under the European Union's Seventh Framework Programme FP/2007-2013, ERC [grant number 331024], a Wallenberg Academy Fellowship and the Swedish Foundation for Strategic Research to AA. The funding body had no role in the design, collection and conclusion of this study.

\section{Authors' contributions}

JSE conceived this study with the support from co-authors. JSE carried out all the field and laboratory work and performed all the analyses with support from co-authors. JSE and DJB wrote the code. JSE lead the writing with contributions from all co-authors.

\section{Acknowledgements}

We thank Anna Ansebo, Claes Persson and our research group for advice, discussion, access to resources and materials. The authors would like to acknowledge support from Science for Life Laboratory, the National Genomics Infrastructure, NGI, and Uppmax for providing assistance in massive parallel sequencing and computational infrastructure. The analyses were performed on the bioinformatics computer cluster Albiorix (http://albiorix.bioenv.gu.se/) at the Department of Biological and Environmental Sciences, University of Gothenburg.

\section{Abbreviations:}

Author abbreviations can be found in (Brummitt and Powell 1992).

\section{References}

Bates, D. M., 1969 Generic relationships in the Malvaceae, Tribe Malvaea. Gentes Herb. 10: 117-135.

Bates, D. M., and O. J. Blanchard Jr, 1970 Chromosome numbers in the Malvales. II. New or otherwise noteworthy counts relevant to classification in the Malvaceae, tribe Malveae. American Journal of Botany: 927-934.

Baum, D. A., S. DeWitt Smith, A. Yen, W. S. Alverson, R. Nyffeler et al., 2004 Phylogenetic relationships of Malvatheca (Bombacoideae and Malvoideae; Malvaceae sensu lato) as inferred from plastid DNA sequences. American Journal of Botany 91: 1863-1871.

Blanc, G., and K. H. Wolfe, 2004 Widespread paleopolyploidy in model plant species inferred from age distributions of duplicate genes. The Plant Cell 16: 1667-1678.

Borrill, P., N. Adamski and C. Uauy, 2015 Genomics as the key to unlocking the polyploid potential of wheat. New Phytologist 208: 1008-1022. 
Bouckaert, R., J. Heled, D. Kühnert, T. Vaughan, C.-H. Wu et al., 2014 BEAST 2: a software platform for Bayesian evolutionary analysis. Journal of PLoS Computational Biology 10: e1003537.

Browning, S. R., and B. L. Browning, 2011 Haplotype phasing: existing methods and new developments. Nature Reviews Genetics 12: 703.

Brummitt, P., and C. E. Powell, 1992 Authors of plant names. Royal Botanic Gardens Kew.

Buggs, R. J., S. Renny-Byfield, M. Chester, I. E. Jordon-Thaden, L. F. Viccini et al., 2012 Next-generation sequencing and genome evolution in allopolyploids. American Jjournal of Botany 99: 372-382.

Cannon, S. B., L. Sterck, S. Rombauts, S. Sato, F. Cheung et al., 2006 Legume genome evolution viewed through the Medicago truncatula and Lotus japonicus genomes. Proceedings of the National Academy of Sciences USA 103: 14959-14964.

Dasgupta, A., 1981 Cytotaxonomy of Malvaceae II. Cytologia 46: 149-160.

Davie, J. H., 1933 Cytological studies in the Malvaceae and certain related families. Journal of Genetics 28: 33-67.

Doyle, J. J., 1992 Gene trees and species trees: molecular systematics as one-character taxonomy. Systematic Botany 17: 144-163.

Drummond, A. J., S. Y. Ho, M. J. Phillips and A. Rambaut, 2006 Relaxed phylogenetics and dating with confidence. Journal of PLoS Biology 4.

Drummond, A. J., M. A. Suchard, D. Xie and A. Rambaut, 2012 Bayesian phylogenetics with BEAUti and the BEAST 1.7. Molecular Biology and Evolution 29: 1969-1973.

Fawcett, J. A., S. Maere and Y. Van de Peer, 2009 Plants with double genomes might have had a better chance to survive the Cretaceous-Tertiary extinction event. Proceedings of the National Academy of Sciences USA 106: 5737-5742.

Fernández, A., A. Krapovickas, G. Lavia and G. Seijo, 2003 Cromosomas de Malváceas. Bonplandia: 141145.

Fryxell, P. A., 1999 Pavonia Cavanilles (Malvaceae). Flora Neotropica Monograph 76.

Fu, L., B. Niu, Z. Zhu, S. Wu and W. Li, 2012 CD-HIT: accelerated for clustering the next-generation sequencing data. Bioinformatics 28: 3150-3152.

Grant, V., 1981 Plant Speciation. New York: Columbia University Press.

Gregg, W. T., S. H. Ather and W. M. Hahn, 2017 Gene-tree reconciliation with MUL-trees to resolve polyploidy events. Systematic Biology 66: 1007-1018. 
Huelsenbeck, J. P., B. Larget and M. E. Alfaro, 2004 Bayesian phylogenetic model selection using reversible jump Markov chain Monte Carlo. Molecular biology and evolution 21: 1123-1133.

Jaillon, O., J.-M. Aury, B. Noel, A. Policriti, C. Clepet et al., 2007 The grapevine genome sequence suggests ancestral hexaploidization in major angiosperm phyla. Nature 449: 463.

Jiao, Y., J. Leebens-Mack, S. Ayyampalayam, J. E. Bowers, M. R. McKain et al., 2012 A genome triplication associated with early diversification of the core eudicots. Genome Biology 13: R3.

Johnson, M. T., E. J. Carpenter, Z. Tian, R. Bruskiewich, J. N. Burris et al., 2012 Evaluating methods for isolating total RNA and predicting the success of sequencing phylogenetically diverse plant transcriptomes. PloS One 7: e50226.

Katoh, K., K. Misawa, K. i. Kuma and T. Miyata, 2002 MAFFT: a novel method for rapid multiple sequence alignment based on fast Fourier transform. Journal of Nucleic Acids Research 30: 3059-3066.

Katoh, K., and D. M. Standley, 2013 MAFFT multiple sequence alignment software version 7: improvements in performance and usability. Molecular Biology and Evolution 30: 772-780.

Kay, K. M., J. B. Whittall and S. A. Hodges, 2006 A survey of nuclear ribosomal internal transcribed spacer substitution rates across angiosperms: an approximate molecular clock with life history effects. $B M C$ Evolutionary Biology 6:36.

Kearse, M., R. Moir, A. Wilson, S. Stones-Havas, M. Cheung et al., 2012 Geneious Basic: an integrated and extendable desktop software platform for the organization and analysis of sequence data.

Bioinformatics 28: 1647-1649.

Kim, Y., S. Kim, N. Koo, A. Shin, S. Yeom et al., 2017 Genome analysis of Hibiscus syriacus provides insights of polyploidization and indeterminate flowering in woody plants. DNA Research 24: 71-80.

Koopman, M. M., and D. A. Baum, 2008 Phylogeny and biogeography of tribe Hibisceae (Malvaceae) on Madagascar. Systematic Botany 33: 364-374.

Landis, J. B., Soltis, D. E., Li, Z., Marx, H. E., Barker, M. S., Tank, D. C., and Soltis, P. S.. 2018. Impact of whole-genome duplication events on diversification rates in angiosperms. American Journal of Botany 105: 348- 363.

Lawrence, W. J. C., 1931 The secondary association of chromosomes. Cytologia 2: 352-384.

Les, D. H., 2017 Aquatic dicotyledons of North America: ecology, life history, and systematics. CRC Press.

Li, H., B. Handsaker, A. Wysoker, T. Fennell, J. Ruan et al., 2009 The sequence alignment/map format and SAMtools. Bioinformatics 25: 2078-2079. 
Li, W., and A. Godzik, 2006 Cd-hit: a fast program for clustering and comparing large sets of protein or nucleotide sequences. Bioinformatics 22: 1658-1659.

Marshall, D. C., C. Simon and T. R. Buckley, 2006 Accurate branch length estimation in partitioned Bayesian analyses requires accommodation of among-partition rate variation and attention to branch length priors. Systematic Biology 55: 993-1003.

Matasci, N., L.-H. Hung, Z. Yan, E. J. Carpenter, N. J. Wickett et al., 2014 Data access for the 1,000 Plants (1KP) project. GigaScience 3: 17.

Menzel, M. Y., 1966 The pachytene chromosome complement of Hibiscus cannabinus. Cytologia 31: 3642.

Menzel, M. Y., and F. D. Wilson, 1969 Genetic relationships in Hibiscus sect. Furcaria. Brittonia 21: 91.

Murray, B. G., L. A. Craven and P. J. De Lange, 2008 New observations on chromosome number variation in Hibiscus trionum s.l. (Malvaceae) and their implications for systematics and conservation. New Zealand Journal of Botany 46:315-319.

Otto, S. P., and J. Whitton, 2000 Polyploid incidence and evolution. Annual Review of Genetics 34: 401437.

Paterson, A. H., J. F. Wendel, H. Gundlach, H. Guo, J. Jenkins et al., 2012 Repeated polyploidization of Gossypium genomes and the evolution of spinnable cotton fibres. Nature 492: 423.

Pellicer, J., O. Hidalgo, S. Dodsworth and I. J. Leitch, 2018 Genome size diversity and its impact on the evolution of land plants. Genes 9: 88.

Pfeil, B., C. Brubaker, L. Craven and M. Crisp, 2002 Phylogeny of Hibiscus and the tribe Hibisceae (Malvaceae) using chloroplast DNA sequences of ndhF and the rpl16 intron. Systematic Botany: 333-350.

Pfeil, B., C. L. Brubaker, L. A. Craven and M. Crisp, 2004 Paralogy and orthology in the Malvaceae rpb2 gene family: investigation of gene duplication in Hibiscus. Molecular Biology and Evolution 21: 14281437.

Pfeil, B., and M. Crisp, 2005 What to do with Hibiscus? A proposed nomenclatural resolution for a large and well known genus of Malvaceae and comments on paraphyly. Australian Systematic Botany 18: 4960 .

Rabier, C.-E., T. Ta and C. Ané, 2013 Detecting and locating whole genome duplications on a phylogeny: a probabilistic approach. Molecular Biology Evolution 31: 750-762.

Ronquist, F., and J. P. Huelsenbeck, 2003 MrBayes 3: Bayesian phylogenetic inference under mixed models. Bioinformatics 19: 1572-1574. 
Schnable J. C., Springer N. M., Freeling M., 2011 Differentiation of the maize subgenomes by genome dominance and both ancient and ongoing gene loss. Proceedings of the National Academy of Sciences USA 108: 4069-4074.

Schranz, M. E., and T. Mitchell-Olds, 2006 Independent ancient polyploidy events in the sister families Brassicaceae and Cleomaceae. The Plant Cell 18:1152-1165.

Seelanan, T., A. Schnabel and J. F. Wendel, 1997 Congruence and consensus in the cotton tribe (Malvaceae). Systematic Botany 22: 259-290.

Skovsted, A., 1941 Chromosome numbers in the Malvaceae II. Comptes rendus des traveaux du laboratoire Carlberg. Physiologique: 195-242.

Soltis, D. E., V. A. Albert, J. Leebens-Mack, C. D. Bell, A. H. Paterson et al., 2009 Polyploidy and angiosperm diversification. American Journal of Botany 96: 336-348.

Soltis, P. S., D. B. Marchant, Y. Van de Peer and D. E. Soltis, 2015 Polyploidy and genome evolution in plants. Current Opinion in Genetics \& Development 35: 119-125.

Stebbins, G., 1947 Types of polyploids: their classification and significance. Advances in GGenetics 1: 403-429.

Stebbins Jr, C., 1950 Variation and evolution in plants: progress during the past twenty years. in Essays in Evolution and Genetics in Honor of Theodosius Dobzhansky, edited by M. K. Hecht and W. C. Steere. Springer, Boston, MA.

Tamura, K., and M. Nei, 1993 Estimation of the number of nucleotide substitutions in the control region of mitochondrial DNA in humans and chimpanzees. Molecular Biology and Evolution 10: 512-526.

Tang, H., X. Wang, J. E. Bowers, R. Ming, M. Alam et al., 2008 Unraveling ancient hexaploidy through multiply-aligned angiosperm gene maps. Genome Research: gr. 080978.080108.

Thomas BC, Pedersen B, Freeling M. 2006 Following tetraploidy in an Arabidopsis ancestor, genes were removed preferentially from one homeolog leaving clusters enriched in dose-sensitive genes. Genome Research 16:934-46.

Turner, B. L., and M. G. Mendenhall, 1993 A revision of Malvaviscus (Malvaceae). Annals of the Missouri Botanical Garden: 439-457.

Tuskan, G. A., S. Difazio, S. Jansson, J. Bohlmann, I. Grigoriev et al., 2006 The genome of black cottonwood, Populus trichocarpa (Torr. \& Gray). Science 313: 1596-1604.

Wagenmakers, E. J., and S. Farrell, 2004 AIC model selection using Akaike weights. Psychonomic Bulletin 11:192-196. 
Wang, K., Z. Wang, F. Li, W. Ye, J. Wang et al., 2012 The draft genome of a diploid cotton Gossypium raimondii. Nature Genetics 44: 1098.

Vanneste, K., Y. Van de Peer and S. Maere, 2012 Inference of genome duplications from age distributions revisited. Molecular Biology and Evolution 30: 177-190.

Wanscher, J., 1934 The basic chromosome number of the higher plants. New Phytologist 33: 101-126.

Wendel, J. F., 2015 The wondrous cycles of polyploidy in plants. American Journal of Botany 102: 17531756.

Wendel, J. F., and R. C. Cronn, 2003 Polyploidy and the evolutionary history of cotton. Advances in Agronomy 78: 139.

Wendel, J. F., A. Schnabel and T. Seelanan, 1995 An unusual ribosomal DNA sequence from Gossypium gossypioides reveals ancient, cryptic, intergenomic introgression. Molecular Phylogenetics and Evolution 4: 298-313.

Wickett, N. J., S. Mirarab, N. Nguyen, T. Warnow, E. Carpenter et al., 2014 Phylotranscriptomic analysis of the origin and early diversification of land plants. Proceedings of the National Academy of Sciences USA 111: E4859-E4868.

Wilson, F. D., 1994 The genome biogeography of Hibiscus L. section Furcaria DC. Genetic Resources and Crop Evolution 41: 13-25.

Wilson, F. D., 2006 A distributional and cytological survey of the presently recognized taxa of Hibiscus section Furcaria (Malvaceae). Bonplandia: 53-62.

Wood, T. E., N. Takebayashi, M. S. Barker, I. Mayrose, P. B. Greenspoon et al., 2009 The frequency of polyploid speciation in vascular plants. Proceedings of the National Academy of Sciences USA 106: 13875-13879.

Woodhouse MR, Schnable JC, Pedersen BS, Lyons E, Lisch D, Subramaniam S, Freeling M. 2010 Following tetraploidy in maize, a short deletion mechanism removed genes preferentially from one of the two homologs. PLoS Biol. 8:e1000409.

Xie, Y., G. Wu, J. Tang, R. Luo, J. Patterson et al., 2014 SOAPdenovo-Trans: de novo transcriptome assembly with short RNA-Seq reads. Bioinformatics 30: 1660-1666.

Xu, Q., G. Xiong, P. Li, F. He, Y. Huang et al., 2013 Correction: Analysis of Complete Nucleotide Sequences of 12 Gossypium Chloroplast Genomes: Origin and Evolution of Allotetraploids. PloS One 8.

Zheng Li, Michael S Barker, Inferring putative ancient whole-genome duplications in the 1000 Plants (1KP) initiative: access to gene family phylogenies and age distributions, GigaScience 9:giaa004. 
Zhang, Y., G.-h. Xu, X.-y. Guo and L.j. Fan, 2005 Two ancient rounds of polyploidy in rice genome. Journal of Zhejiang University. Science. B 6: 87-90.

\section{Additional Files}

Additional file 1: Table 1_species accession.docx

Species name and accession information

BONN stand for Botanische Gärten der Universität Bonn; The Royal Botanic Gardens, Kew; USDA National Plant Germplasm System. Hibiscus trionum is kept as a cultivar at Christchurch Botanic Gardens (NZ). Number of reads after quality trimming, in parenthesis the percentage of reduced reads from the original raw data. Percent of GC content after the trimming. Percentage of recovered loci per species and the total number of loci. Percentage of recovered probes (designed over exons) per species. Vouchers are deposited at the Gothenburg herbarium (GB).

Supplementary fig. 1. Phylogenetic relationships of a single-copy gene (SCG) Oxysterol-D1 inferred by MrBayes.

Supplementary figs. 2-21. MrBayes trees of multi-copy genes (MSC).

\section{Figures}


S1:Two known WGDs on branch leading to S

S2: One WGD on branch leading to $S$, and one WGD shared by $(\mathrm{S}, \mathrm{T}, \mathrm{P}, \mathrm{C}, \mathrm{M})$

S3: Two WGDs shared by (S, T, P, C, M)
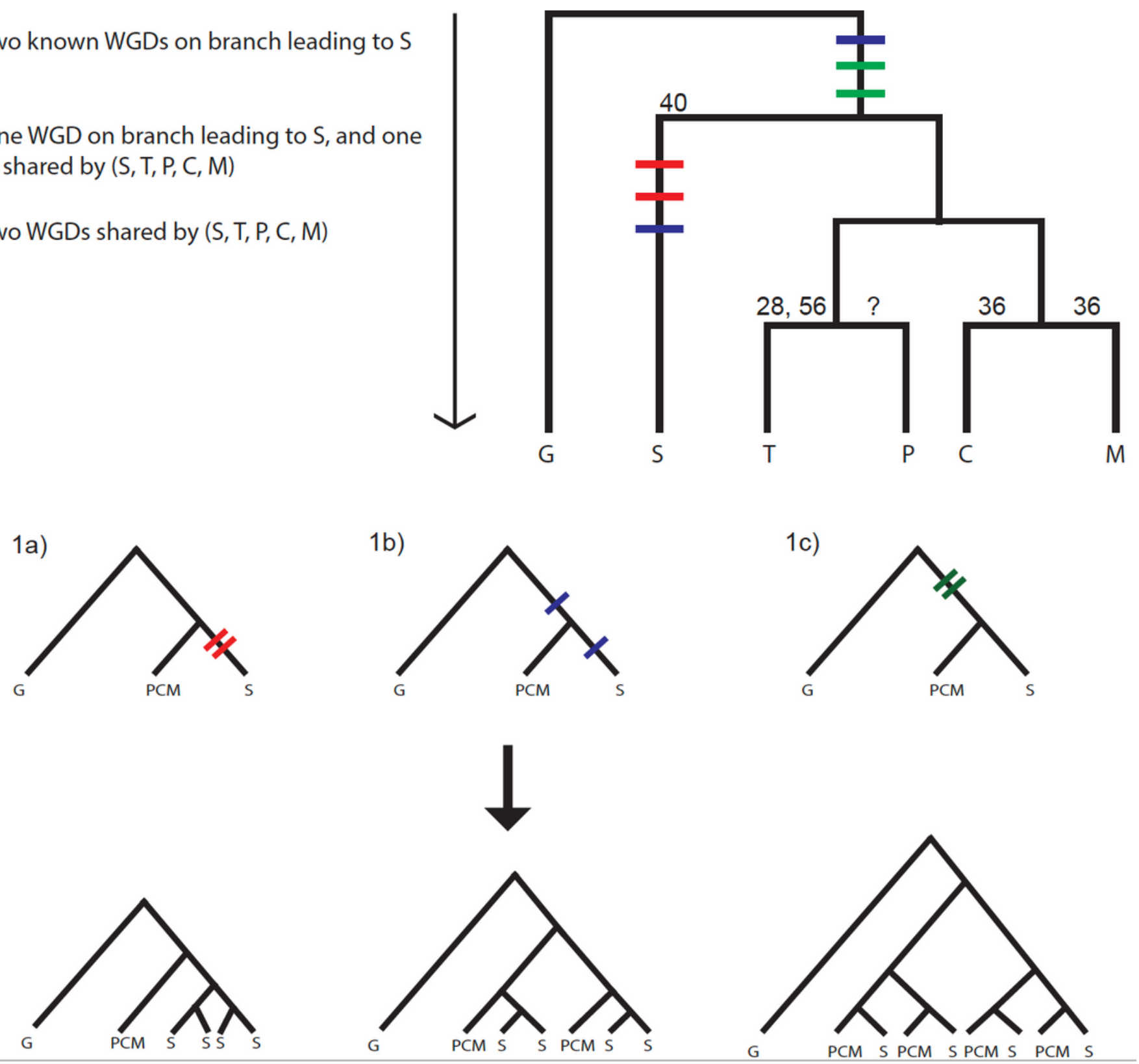

Figure 1

Three hypothetical genome evolution scenarios in Hibiscus with Gossypium raimondii (G) as outgroup. S is short for for $\mathrm{H}$. syriacus; $\mathrm{T}$ for $\mathrm{H}$. trionum; $\mathrm{P}$ for Pavonia triloba; $\mathrm{C}$ for $\mathrm{H}$. cannabinus; and $\mathrm{M}$ for $\mathrm{H}$. mechowii. The different colors represent three different genome duplication scenarios, $1 \mathrm{a}$ ) where red = S1 (two independent WGD in S), 1b) blue = S2 (one WGD shared by all species of Hibiscus and one independent in S) and 1c) green = S3 (two WGD events shared by all species of Hibiscus). Numbers above branches leading to each species are known chromosome counts. 
S1:Two known WGDs on branch leading to $S$

S2: One WGD on branch leading to $S$, and one WGD shared by (S, T, P, C, M)

S3: Two WGDs shared by (S, T, P, C, M)

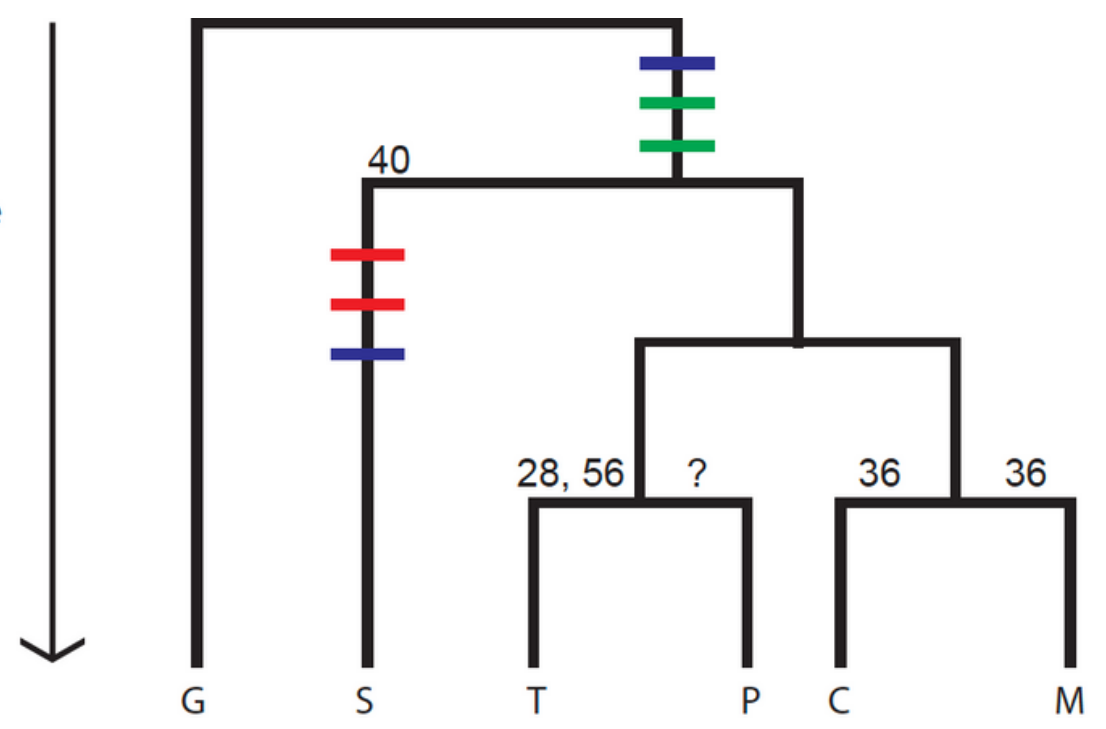

1b)
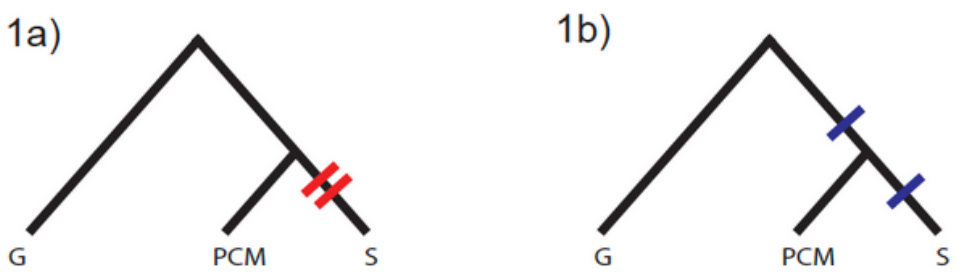

1c)
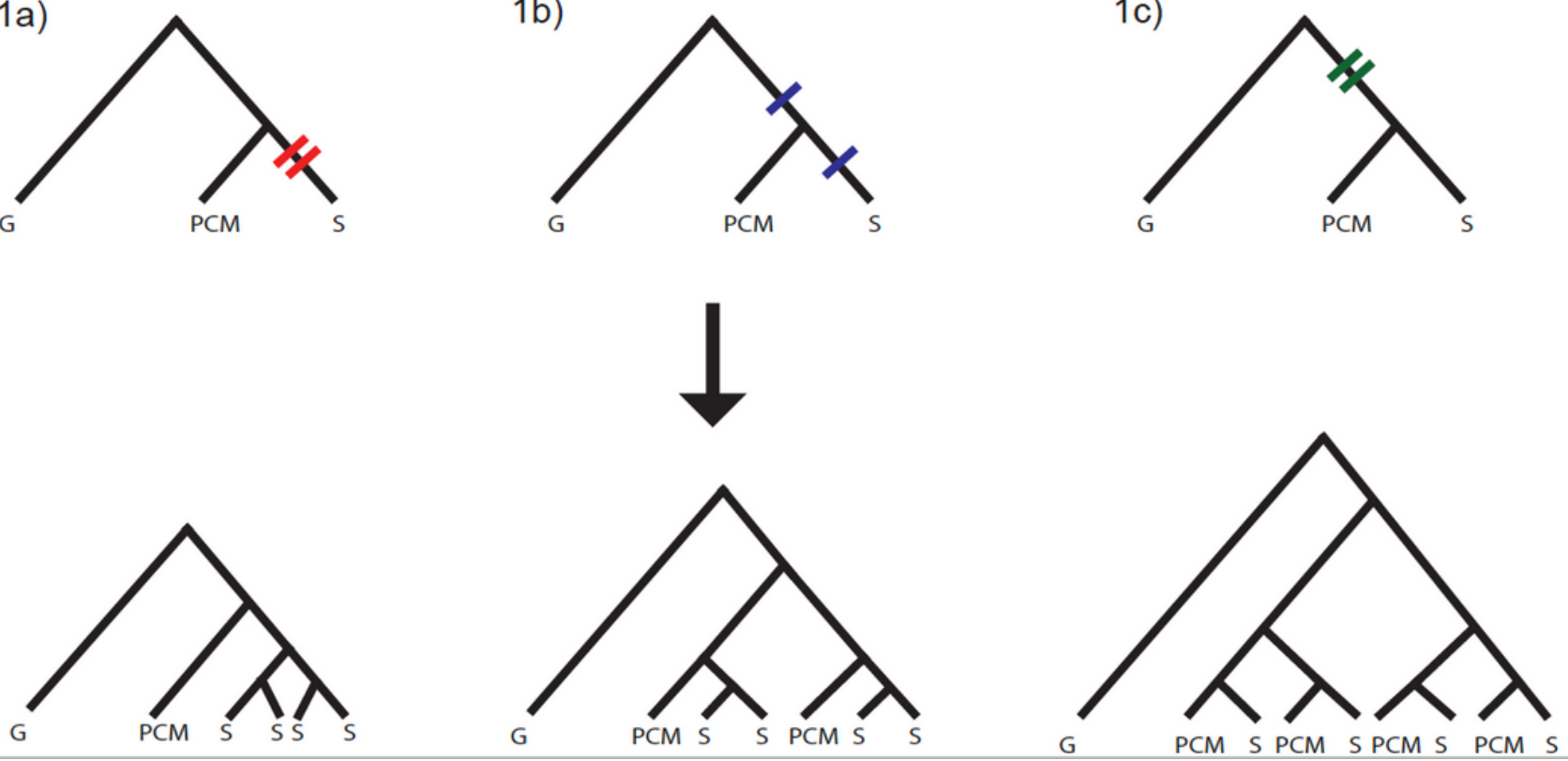

\section{Figure 1}

Three hypothetical genome evolution scenarios in Hibiscus with Gossypium raimondii (G) as outgroup. S is short for for $\mathrm{H}$. syriacus; $\mathrm{T}$ for $\mathrm{H}$. trionum; $\mathrm{P}$ for Pavonia triloba; $\mathrm{C}$ for $\mathrm{H}$. cannabinus; and $\mathrm{M}$ for $\mathrm{H}$. mechowii. The different colors represent three different genome duplication scenarios, $1 \mathrm{a}$ ) where red = S1 (two independent WGD in S), 1b) blue = S2 (one WGD shared by all species of Hibiscus and one independent in S) and 1c) green = S3 (two WGD events shared by all species of Hibiscus). Numbers above branches leading to each species are known chromosome counts. 


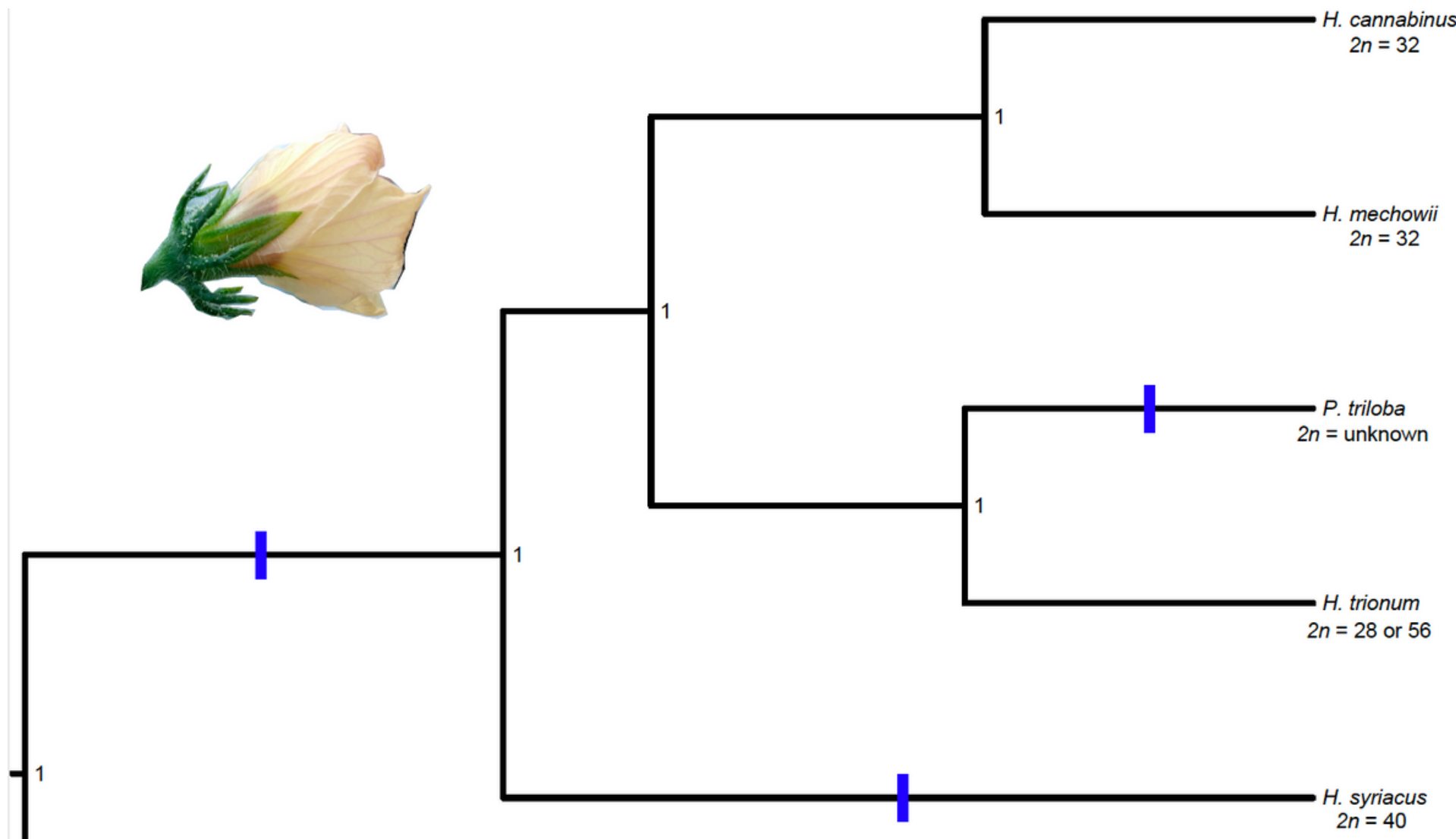

Figure 2

Species tree inferred by StarBEAST using 10 single-copy genes. The blue color indicates the most probable placement of the whole-genome duplication events. Supplementary fig. 1. Phylogenetic relationships of a single-copy gene (SCG) Oxysterol-D1 inferred by MrBayes. 


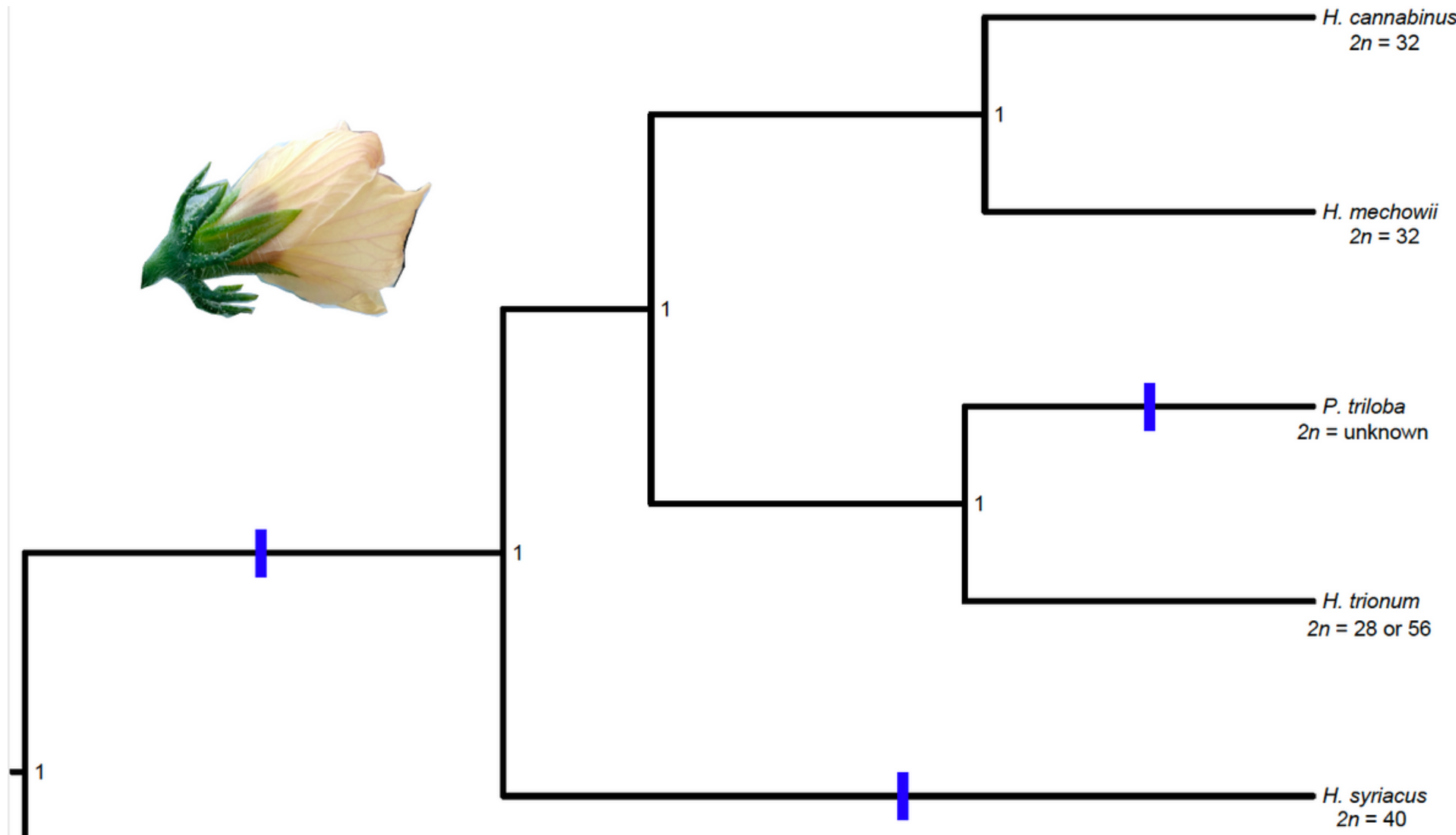

Figure 2

Species tree inferred by StarBEAST using 10 single-copy genes. The blue color indicates the most probable placement of the whole-genome duplication events. Supplementary fig. 1. Phylogenetic relationships of a single-copy gene (SCG) Oxysterol-D1 inferred by MrBayes.

\section{Supplementary Files}

This is a list of supplementary files associated with this preprint. Click to download.

- Table1speciesaccession.docx

- Table1speciesaccession.docx

- Supplfigure1.pdf

- Supplfigure1.pdf

- Supplfigure2ABCC2v4.pdf

- Supplfigure2ABCC2v4.pdf

- Supplfigure3ACCSv4.pdf 
- Supplfigure3ACCSv4.pdf

- Supplfigure4Acylaminov2.pdf

- Supplfigure4Acylaminov2.pdf

- Supplfigure5AglucanPv3.pdf

- Supplfigure5AglucanPv3.pdf

- Supplfigure6Ankyrinv5.pdf

- Supplfigure6Ankyrinv5.pdf

- Supplfigure7Bgalactosidase8v3.pdf

- Supplfigure7Bgalactosidase8v3.pdf

- Supplfigure8CADv3.pdf

- Supplfigure8CADv3.pdf

- Supplfigure9Calcium11v3.pdf

- Supplfigure9Calcium11v3.pdf

- Supplfigure10CalciumATPase1v4.pdf

- Supplfigure10CalciumATPase1v4.pdf

- Supplfigure11CesA1v4.pdf

- Supplfigure11CesA1v4.pdf

- Supplfigure12EIF2Bv3.pdf

- Supplfigure12EIF2Bv3.pdf

- Supplfigure13F5Hv3.pdf

- Supplfigure13F5Hv3.pdf

- Supplfigure14Formin2v4.pdf

- Supplfigure14Formin2v4.pdf

- Supplfigure15glutaminev3.pdf

- Supplfigure15glutaminev3.pdf

- Supplfigure16GPDHv3.pdf

- Supplfigure16GPDHv3.pdf

- Supplfigure17KinesinKp1v3.pdf

- Supplfigure17KinesinKp1v3.pdf

- Supplfigure18LOC105792102v3.pdf

- Supplfigure18LOC105792102v3.pdf

- Supplfigure19MAP3Kv3.pdf

- Supplfigure19MAP3Kv3.pdf

- Supplfigure20Phospholipasev3.pdf 
- Supplfigure20Phospholipasev3.pdf

- Supplfigure21PlasmaATPasev4.pdf

- Supplfigure21PlasmaATPasev4.pdf 